\title{
Quantitative Messungen über den Elementarprozeß der Lichtanregung von Leuchtstoffen durch einzelne $a$-Teilchen I
}

\author{
Von Immanuel Broser, Hartmut Kallmann und Claus Reuber \\ Aus dem Kaiser-Wilhelm-Institut für physikalische Chemie und Elektrochemie, Berlin-Dahlem \\ (Z. Naturforschg. 5a, 79-85 [1950]; eingegangen am 17. Mai 1949)
}

\begin{abstract}
Es werden die von einzelnen $\alpha$-Teilchen an verschiedenen Leuchtstoffen hervorgerufenen Szintillationen in bezug auf Zeitdauer und Form des Abklingens näher untersucht. Hierzu werden Berechnungen über die Veränderung der den Vervielfacher verlassenden Primärimpulse durch die Zeitkonstante der Meßanordnung angestellt und besonders die physikalisch interessierenden Fälle eines exponentiellen oder hyperbolischen Abklingens diskutiert. Hierbei ergibt sich, daß durch Messung der Impulsmaxima bei verschiedenen Zeitkonstanten der Meßanordnung auf Dauer und Form des Abklingens geschlossen werden kann. Die untersuchten Lichtblitze lassen sich allgemein durch einen exponentiellen Abfall mit Zeitkonstanten zwischen 0,2 und $3,7 \cdot 10^{6} \mathrm{sec}^{-1}$ beschreiben. Auch stark phosphoreszierende Leuchtstoffe erscheinen zur $\alpha$-Teilchen-Zählung geeignet. Um einen sinnvollen Anschluß an Messungen mit großer Teilchenzahl zu gewinnen, muß angenommen werden, daß außer den sehr kurzen Lichtblitzen noch eine wesentlich längere „momentane Phosphoreszenz“ und danach erst die eigentliche Phosphoreszenz auftritt.
\end{abstract}

E ist seit langem bekannt, daß $\alpha$-Teilchen beim Auftreffen auf Leuchtstoffe Lichtblitze erzeugen, die - je nach Art des untersuchten, lumineszenzfähigen Stoffes - mehr oder minder gut mit dem dunkel adaptierten Auge zu erkennen sind. Dieser Effekt bildete die Grundlage für eine früher viel verwendete Methode zum Nachweis einzelner $\alpha$-Teilchen, die jedoch infolge einer Reihe von Unzulänglichkeiten später durch elektrische Nachweismethoden ersetzt wurde. Die Nachteile dieser sog. „Szintillationsmethode" beruhen weniger auf unerwünschten Eigenschaften des Leuchtprozesses als vielmehr darauf, daß zur Zählung der Szintillationen das menschliche Auge benutzt wurde. Gemessen werden konnte meist erst nach einer längeren Adaptionszeit, die Zahl der registrierbaren $\alpha$-Teilchen war nach unten und oben stark begrenzt, und außerdem war der Meßfehler ziemlich groß. Aus dem gleichen Grunde war auch die Erforschung der physikalischen Eigenschaften des Szintillationsprozesses erschwert. Man wußte nur wenig über die absolute Lichtausbeute - das Verhältnis der ausgesandten Lichtenergie zur eingestrahlten, absorbierten $\alpha$-Energie - sowie über die Dauer des Lichtblitzes und eine Angabe über die Form der Abklingung des Leuchtens konnte erst recht nicht gemacht werden.

Diesen Schwierigkeiten wurde in neuerer Zeit dadurch begegnet, daß an Stelle des menschlichen Auges eine hochempfindliche Photozelle mit Sekundärelektronenvervielfacher zur Ausmessung der Lichtblitze verwendet wurde ${ }^{1,2}$. Es gelang nicht nur zu zeigen, daß die Leuchtstoffmethode zur Registrierung von $\alpha$-Teilchen und anderen energiereichen Korpuskularteilchen sowie von $\gamma$ - und Höhenstrahlen eine Reihe von Vorteilen gegenüber den bisherigen elektrischen Nachweismethoden besitzt ${ }^{3}$, sondern es war auch möglich, den Einzelprozeß der Anregung von Leuchtstoffen mit den genannten Strahlenarten genau zu analysieren. Besonders an $a$-Teilchen wurden derartige Messungen durchgeführt: So konnte gezeigt werden, daß die Lichterzeugung bei Einkristallen (insbesondere aus CdS) streng proportional der Energie der $\alpha$-Teilchen erfolgt und daß auch in polykristallinem Material auf gleiche absorbierte Energie vieler $\alpha$-Teilchen eine gleich große emittierte Lichtenergie kommt ${ }^{4}$. Auch die Zeitdauer von $\alpha$-Szintillationen konnte - jedenfalls annähernd - angegeben werden. So fanden wir bei $\mathrm{ZnS}$ eine Abklingzeit von etwa $10^{-6}$ sec und bei Naphthalin von $\approx 10^{-7}$ sec. Die Genauigkeit dieser Messungen war aber nicht erheblich, und es konnte nichts über die Form der Abklingung des Leuchtens ausgesagt werden. Außerdem waren nur wenige Substanzen untersucht worden.

1 I. Broser u. H. Kallmann, Z. Naturforschg. 2a, 439 [1947].

2 I. Broser u. H. Kallma nn, Z. Naturforschg. 2a, 642 [1947].

3 I. B roser, L. Herforth, H. Ka llmann u. U. M. M a rtius, Z. Naturforschg. 3a, 6 [1948].

4 I. Broser u. H. Kallmann, Ann. Physik 3, 317 [1948]; 4, 61 [1948]. 


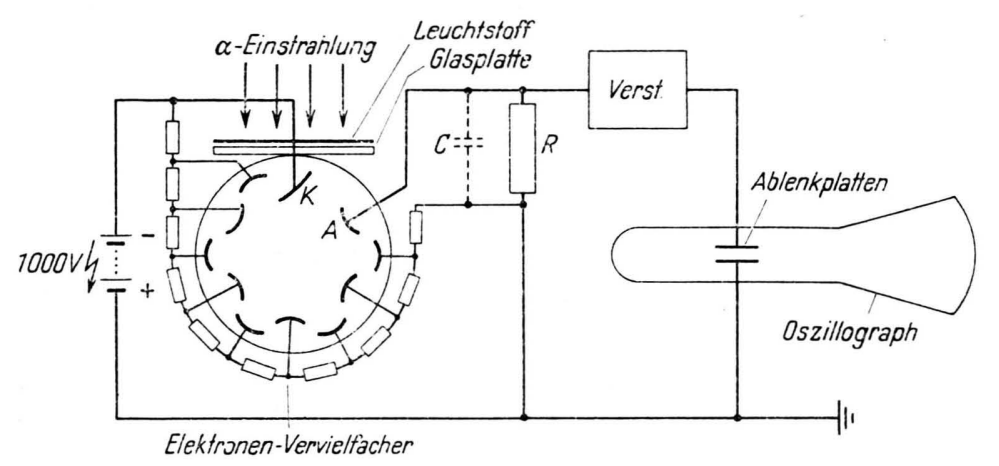

Abb. 1. Meßanordnung.

Im folgenden werden Methoden angegeben, mit denen es gelingt, außer der genauen Zeitdauer der Lichtblitze auch die Form der Abklingkurven zu bestimmen. Messungen dieser Art an 13 verschiedenen Leuchtstoffen werden beschrieben und die Abklingzeiten und -gesetze bei diesen Substanzen angegeben.

\section{Meß anordnung}

Die Beobachtung der Lichtblitze erfolgte in einer Anordnung entsprechend der Abb. 1.

Die $\alpha$-Teilchen traten aus einem Poloniumpräparat geringer Aktivität aus und gelangten in den zu untersuchenden, in dünner Schicht auf einer Glasplatte aufgetragenen Leuchtstoff, wo sie einzelne Szintillationen erzeugten. Das Lumineszenzlicht löste aus der Photokathode eines Sekundärelektronenvervielfachers (Typ 931A) einen Elektronenstrom aus, der im Vervielfachersystem etwa um einen Faktor $10^{6}$ verstärkt wurde. Der Ausgangsstrom erzeugte am Arbeitswiderstand $\mathrm{R}$ einen Spannungsabfall, der mit Hilfe eines einfachen Verstärkers noch etwas vergrößert wurde und auf dem Oszillographenschirm einen Impulsstrich erzeugte, dessen Länge leicht auszumessen war.

Die zeitliche Änderung der Spannung an den Oszillographenplatten ist nicht die gleiche wie die zeitliche Änderung der Intensität des Primärlichtblitzes. Zwar arbeiten der Elektronen-Vervielfacher bis zu Frequenzen von $10^{7} \mathrm{~Hz}$ und der Verstärker des Oszillographen bis zu Frequenzen von $10^{\circ} \mathrm{Hz}$ verzerrungsfrei, aber durch Parallelkapazitäten des Arbeitswiderstandes wird die Impulsform verändert. Trotzdem ist es möglich, auf Grund des Oszillographenbildes den Szintillationsvorgang zu analysieren und Rückschlüsse auf Dauer, Intensität und Form der Abklingkurve zu ziehen.

\section{Die Veränderung des Primär-}

lichtblitzes durch die Meßanordnung

Betrachten wir die Übertragung des Impulses vom Vervielfacherausgang auf das Gitter der ersten Verstärkerröhre des Oszillographenverstärkers. Sie findet mit Hilfe einer großen Kopplungskapazität statt
$(0,5 \mu \mathrm{F})$, die für die sehr kurzzeitigen Impulse praktisch als Kurzschluß wirkt. Im Prinzipschaltbild Abb. 2 ist sie daher nicht mehr eingezeichnet. Die oben erwähnte Parallelkapazität $C$ setzt sich somit nur aus Kapazitäten der abgeschirmten Leitungen und Kapazitäten der ersten Verstärkerröhre zusammen. Dem

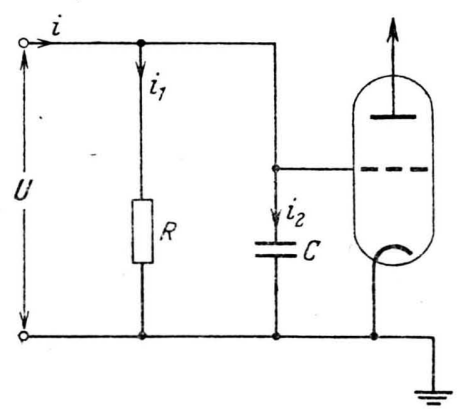

Abb. 2. Prinzipschaltbild des $R C$-Gliedes.

Außenwiderstand des Vervielfachers ist der Gitterableitwiderstand der Verstärkerröhre (1,5 M $\Omega$ ) parallel geschaltet, so daß beide zusammen die Rolle des Arbeitswiderstandes $R$ übernehmen.

Gelangt in die abgebildete Schaltung ein Stromimpuls, so verteilt sich der Strom auf Kondensator $C$ und Arbeitswiderstand $R$ und erzeugt einen Spannungsimpuls:

$$
\begin{array}{r}
U=i_{1} R=1 / C \cdot \int_{0}^{t} i_{2} d t=1 / C \cdot \int_{0}^{t}\left(i-i_{1}\right) d t \\
=1 / C \cdot \int_{0}^{t}(i-U / R) d t .
\end{array}
$$

Dies führt zu einer Differentialgleichung für $U$ :

$$
d U / d t+U / R C=i / C
$$

mit der Lösung: 


$$
U=1 / C \cdot e^{-\varphi t} \int_{0}^{t} i e^{\Upsilon t} d t
$$

wobei

$$
\varphi=1 / R C
$$

die Zeitkonstante der Anordnung darstellt.

Um Aussagen über den Spannungsverlauf $U$ machen zu können, muß der Stromverlauf $i$ bekannt sein. Da dieser aber dem zeitlichen Verlauf des Leuchtens folgt, kann für ihn nur ein exponentieller oder hyperbolischer Abfall in Frage kommen. Bei ersterem ist die Zeitkonstante zu bestimmen, bei dem zweiten eine unten näher definierte Konstante.

Behandeln wir zunächst den Fall der exponentiellen Abklingung. Da die Zeit, die zur Erregung des Leuchtstoffes durch ein $\alpha$-Teilchen gebraucht wird, sicher etwa gleich der Lebensdauer dieses Teilchens, also klein gegen die Abklingzeit des Leuchtens ist, braucht ein etwaiger Anklingvorgang nicht berücksichtigt zu werden, und wir können den durch den Leuchtvorgang verursachten Stromimpuls im $R C$ Glied beschreiben durch:

$$
i=i_{0} e^{-c t t},
$$

wobei $\alpha$ die Zeitkonstante des Leuchtprozesses, also die zu bestimmende Größe, darstellt. Nach Einsetzen von (5) in (3) ergibt die Rechnung:

$$
U=\frac{i_{0}}{C} \frac{1}{\varphi-c}\left\{e^{-a t}-e^{-\varphi t}\right\} .
$$

Betrachten wir diese Gleichung, so sehen wir, daß der dem zeitlichen Verlauf von $U$ proportionale Vorgang auf dem Oszillographenschirm im Ansteigen des Ausschlages bis zu einem Maximum und darauf folgendem Abklingen besteht, das je nach dem Verhältnis von $\alpha \mathrm{zu} \varphi$ entweder dem ursprünglichen Lichtabklingen oder aber einem dem $R C$-Gliede entsprechenden Abklingvorgang folgt. Das der Messung am leichtesten zugängliche Maximum (Länge $l$ des Impulsstriches auf dem Oszillographenschirm) ergibt sich nach Differentiation von Gl. (6) zu:

$$
l \sim U_{\max }=\frac{i_{0}}{C a}\left(\frac{\varphi}{a}\right)^{[\varphi / a] /[1-(\varphi / c)]},
$$

wobei $i_{0} / \alpha$ die gesamte Ladungsmenge des durch den Lichtblitz verursachten Stromimpulses nach Gl. (5) darstellt.

Da sich sowohl $l$ - als mittlere Länge vieler Impulsstriche - als auch $\varphi=1 / R C$ experimentell leicht bestimmen lassen, kann man durch Variation von $\psi$ (am besten durch Änderung von $R$ ) und Beobachtung der Änderung von $l$ aus zwei Wertepaaren die Größe $\alpha$ errechnen. Es gilt für ein Wertepaar $\left(\varphi_{1}, \varphi_{2}\right)$ nach Gl. (7):

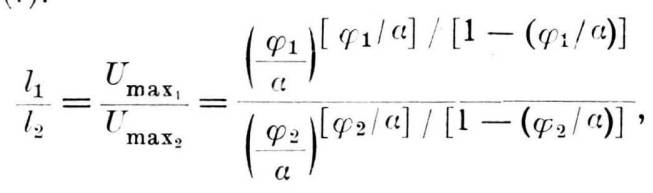

woraus sich die Unbekannte $\alpha-$ am besten auf graphischem Wege - bestimmen läßt. Verwendet man mehr als zwei Werte von $\varphi$, so lassen sich etwaige Abweichungen von der Voraussetzung des exponentiellen Abklingens der Szintillation erkennen.

Bei hyperbolischem Ab̉klingen kann man für den Stromverlauf im Impulsabfall folgenden allgemeinen Ausdruck ansetzen:

$$
i=b /(t+a)^{2} .
$$

Führen wir in diese Gleichung für $t=0$ wie oben den Wert $i_{0}$ ein, so läßt sich Gl. (9) schreiben:

$$
i=i_{0}(t / a+1)^{2} .
$$

Hierin ist $a$ die zu bestimmende, von Stoff zu Stoff verschiedene Größe, von deren Wert die Steilheit des Impulsabfalls abhängt. Für die Lösung der Differentialgleichung ergibt sich durch Einsetzen:

$$
U=\frac{i_{0}}{C} e^{-\varphi t} \int_{0}^{t} \frac{e^{\varphi t}}{(t / c t+1)^{2}} d t .
$$

Aus der Maximumbedingung folgt die Gleichung für die Zeit der Impulsspitze $t_{\max }$ :

$$
\int_{0}^{t_{\max }} \frac{e^{\varphi t}}{(t / a+1)^{2}} d t=\frac{e^{\varphi t_{\max }}}{\varphi\left(t_{\max } / a+1\right)^{2}} .
$$

Aus dieser Gleichung läßt sich auf graphischem Wege ein Zusammenhang zwischen $t_{\max }$ und $a$ für vorgegebenes $\varphi$ erhalten. Durch Einsetzen von Gl. (12) in Gl. (11) ergibt sich für den maximalen Wert des Impulses:

$$
U_{\max }=\frac{i_{0}}{C} \frac{1}{\varphi\left(t_{\max } / a+1\right)^{2}} .
$$

Entsprechend Gl. (8) läßt sich auch im hyperbolischen Fall aus einem Wertepaar $\left(\varphi_{1}, \varphi_{2}\right)$ die unbekannte gesuchte Größe $a$ bestimmen:

$$
\frac{l_{1}}{l_{2}}=\frac{U_{\max _{1}}}{U_{\max _{2}}}=\frac{\varphi_{2}}{\varphi_{1}}\left\{\frac{\left(t_{\max _{2}} / a+1\right)}{\left(t_{\max _{1}} / a+1\right)}\right\}^{2} .
$$


Da jedoch nach Gl. (12) die Werte von $t_{\max }$ in ihrer Abhängigkeit von $a$ nur als Kurven vorliegen, muß das gesuchte $a$ in diesem Fall wiederum auf graphische Weise bestimmt werden.

\section{Die Bestimmung der Abklingzeit von $\mathrm{CaWO}_{4}$}

Als Beispiel für unsere Messungen, die an einer Reihe verschiedenartiger Leuchtstoffe durchgeführt wurden, sei die Bestimmung der Abklingkonstanten des $\mathrm{CaWO}_{4}$ näher beschrieben. Die Messung der Maximalamplitude der durch die im $\mathrm{CaWO}_{4}$ von $\alpha$-Teilchen erregten Lichtblitze erzeugten Spannungsimpulse erfolgte visuell auf dem Oszillographenschirm. Es wurde bei einem bestimmten Außenwiderstand $R$ die Länge $l_{1}$ bestimmt, die von einer

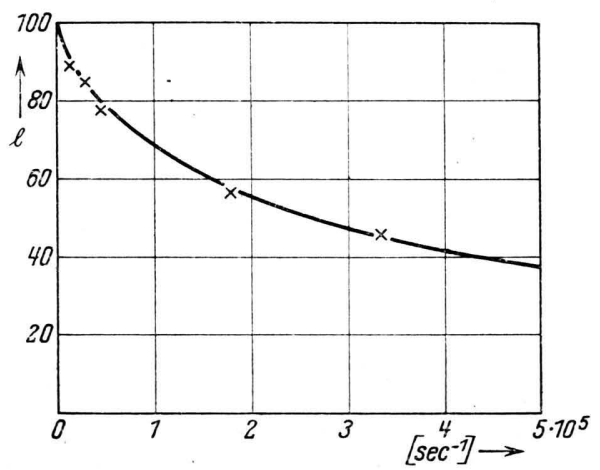

Abb. 3. Impulslänge $l$ (in willkürlichen Einheiten) als Funktion von $\varphi$ für $\mathrm{CaWO}_{4}$.

bestimmten Zahl $A$ von (bei den meisten Leuchtstoffen unterschiedlich langen ${ }^{4}$ ) Impulsstrichen erreicht oder übertroffen wurde. Veränderte man den Außenwiderstand $R$, so änderte sich auch die (mittlere) Länge der Impulse. Als Impulslänge $l_{2}$ bei verändertem Widerstand wurde nun wiederum die Länge ermittelt, die von A Impulsen erreicht oder übertroffen wurde. In dieser Weise wurden 5 verschiedene Außenwiderstände zur Änderung des $R C$-Gliedes benutzt, während die Kapazität der Anordnung konstant $C=6 \cdot 10^{-11} \mathrm{~F}$ gehalten wurde. Die Werte für $\varphi=1 / R C$ ergaben sich dann zu:

$$
\begin{gathered}
\varphi_{1}=1,33 \cdot 10^{4} \mathrm{sec}^{-1} ; \quad \varphi_{2}=2,78 \cdot 10^{4} \mathrm{sec}^{-1} \\
\varphi_{3}=4,40 \cdot 10^{4} \mathrm{sec}^{-1} ; \quad \varphi_{4}=1,06 \cdot 10^{5} \mathrm{sec}^{-1} \\
\varphi_{5}=3,30 \cdot 10^{5} \mathrm{sec}^{-1} .
\end{gathered}
$$

Entsprechend der oben beschriebenen Methode haben wir nach Gl. (8) aus je zwei Meßwerten die Zeitkonstante $\alpha$ ermittelt und als Mittel erhalten:

$$
\alpha=0,53 \cdot 10^{6} \mathrm{sec}^{-1},
$$

d. h. in $1 /\left(0,53 \cdot 10^{6}\right) \mathrm{sec} \approx 2 \cdot 10^{-6} \mathrm{sec}$ ist die Helligkeit des Lichtblitzes auf den $e$-ten Teil abgesunken. Wir haben in die Abb. 3 den nach Gl. (7) errechneten Verlauf der Abnahme von $U_{\max }$ mit zunehmendem $\varphi$ eingezeichnet, wobei $i_{0} /(C \cdot a)$ willkürlich gleich 100 gesetzt wurde.
Die Meßpunkte, die Mittelwerte aus je drei Messungen darstellen, wurden mit einem Faktor normiert in Abb. 3 eingetragen. Man erkennt, daß der Verlauf der Kurve in guter Übereinstimmung mit den tatsächlichen Verhältnissen steht, so daß als sehr wahrscheinlich gelten kann, daß der Abklingvorgang beim $\mathrm{CaWO}_{4}$ einem exponentiellen Gesetz folgt.

\section{Die Bestimmung der Abklingzeit verschiedener Leuchtstoffe}

Nach dem beschriebenen Verfahren wurden 12 weitere Leuchtstoffe durchgemessen und ihre Abklingzeiten ermittelt. Die Untersuchung der Abklingform ergab, daß alle Abklingkurven gut durch ein exponentielles Abklinggesetz beschrieben werden können. Um eine im Rahmen der Meßgenauigkeit vertretbare und allgemeine Beschreibung der Eigenschaften der verschiedenen Leuchtstoffe zu erzielen, haben wir in dieser Arbeit unsere Berechnungen nur noch an Hand der Gln. (7) und (8) im exponentiellen Fall durchgeführt. In Tab. 1 sind in der 2. Spalte die Zeitkonstanten des Abklingens der $\alpha$-Lichtblitze wiedergegeben, die sich praktisch kaum mehr als um eine Zehnerpotenz unterscheiden. Der Wert für CdS, dessen rote Lumineszenz mit dem 931-A-Vervielfacher nicht mehr meßbar war, wurde mit Hilfe eines Weißschen Vervielfachers mit 18 Netzen bei einer Temperatur von etwa $-60^{\circ} \mathrm{C}$ erhalten. Die Messungen an allen anderen Leuchtstoffen wurden bei Zimmertemperatur durchgeführt.

Die Werte der Abklingkonstanten $\alpha$ wurden nach Gl. (8) in voneinander unabhängigen Messungen ermittelt. Wir haben jedoch auch Messungen durchgeführt, um die Verhältnisse der Lichtenergien, die von jedem Leuchtstoff bei Anregung mit einzelnen $\alpha$-Teilchen ausgesandt werden, zu bestimmen.

$\mathrm{Zu}$ diesem Zweck wurden die Leuchtstoffe bei gleicher Fläche und Schichtdicke nacheinander mit dem gleichen $\alpha$-Präparat bestrahlt und die mittlere Länge der Impulse auf dem Oszillographenschirm wieder derart ausgemessen, daß immer die gleiche Anzahl von Impulsstrichen länger war als diese Größe $l$. Das Ergebnis der Messungen ist in der 3. Spalte der Tab. 1 wiedergegeben, wobei die maximale Impulslänge für Naphthalin willkürlich gleich 100 gesetzt wurde. Man erkennt, daß Längenunterschiede bis zu einem Faktor 20 auftreten, muß aber noch berücksichtigen, daß der Vervielfacher 931 A für die verschiedenen Emissionsgebiete der Leuchtstoffe eine unterschiedliche Empfindlichkeit besitzt.

Berechnet man aus diesen Zahlen die Größe der Impulse nach Gl. (7) für $R=\infty$. d. h. für $\varphi=0$, 


\begin{tabular}{|c|c|c|c|c|c|c|c|c|c|}
\hline & Leuchtstoff & $\begin{array}{c}{ }^{(} \\
\sec ^{1} \\
10^{6}\end{array}$ & $\begin{array}{l}l_{\text {gem }} \\
\text { willlk. } \\
\text { Einh. }\end{array}$ & $\begin{array}{l}l_{\infty} \\
\text { willk. } \\
\text { Einh. }\end{array}$ & $\begin{array}{l}J \\
\text { Skt }\end{array}$ & $l_{\infty} / J$ & ") & $\eta_{l}$ & $l_{\text {abs. }}$ \\
\hline $\begin{array}{l}1 \\
2\end{array}$ & $\begin{array}{l}\mathrm{ZnS}-\mathrm{Ag} \ldots \ldots \\
\mathrm{ZnS}-\mathrm{Cu}_{\mathrm{I}} \ldots \ldots \ldots\end{array}$ & $\begin{array}{l}1,87 \\
1,86\end{array}$ & $\begin{array}{r}1880 \\
600\end{array}$ & $\begin{array}{r}1950 \\
622\end{array}$ & $\begin{array}{l}7240 \\
2020\end{array}$ & $\begin{array}{l}0,270 \\
0,310\end{array}$ & $\begin{array}{l}0,280 \\
0,250\end{array}$ & $\begin{array}{l}0,0750 \\
0,0770\end{array}$ & $\begin{array}{l}25,00 \\
25,70\end{array}$ \\
\hline 3 & $\mathrm{ZnS}-\mathrm{Cu}_{\mathrm{II}, \mathrm{n}} \ldots \ldots$ & 2,96 & 735 & 754 & 2950 & 0,255 & 0,140 & 0,0360 & 12,00 \\
\hline 4 & $\mathrm{ZnS}-\mathrm{Cu}_{\mathrm{IU}, \mathrm{Cla}, \mathrm{n}} \ldots$ & 3,70 & 935 & 955 & 3550 & 0,270 & 0,230 & 0,0620 & 20,67 \\
\hline 5 & $\mathrm{CdS}-\mathrm{Ag}^{*} \ldots \ldots$ & 0,20 & $\overline{-}$ & - & - & 0,120 & 0,230 & 0,0280 & 9,33 \\
\hline 6 & $\mathrm{ZnS} / \mathrm{ZnSe}-\mathrm{Ag} \ldots \ldots$ & 0,97 & 553 & 585 & 1500 & 0,390 & 0,120 & 0,0470 & 15,70 \\
\hline 7 & $\mathrm{ZnS} / \mathrm{CdS}-\mathrm{Cu}_{\mathrm{n}} \ldots \ldots$ & 2,04 & 248 & 257 & 704 & 0,370 & 0,120 & 0,0450 & 15,00 \\
\hline 8 & $\mathrm{Zn}_{2} \mathrm{SiO}_{4}-\mathrm{Mn} \ldots \ldots$ & 0,33 & 74 & 84 & $39 \check{5}$ & 0,210 & 0,035 & 0,0073 & 2,44 \\
\hline 9 & $\mathrm{ZnO}-\mathrm{Mn} \ldots \ldots$ & 1,78 & 248 & 257 & 342 & 0,750 & 0,020 & 0,0150 & 5,00 \\
\hline 10 & $\mathrm{CaWO}{ }_{4} \ldots \ldots$ & 0,53 & 263 & 289 & 494 & $0, \overline{85}$ & 0,017 & 0,0100 & 3,33 \\
\hline 11 & $\mathrm{MgWO}$ & 0,81 & 99 & 106 & 135 & 0,785 & 0,017 & 0,0135 & 4,50 \\
\hline 12 & $\mathrm{KBr}-\mathrm{Tl}^{*}$ & 2,00 & 132 & 137 & 396 & 0,345 & 0,017 & 0,0060 & 2,00 \\
\hline 13 & Naphthalin . & 2,00 & 100 & 104 & 104 & 1,000 & 0,003 & 0,0030 & 1,00 \\
\hline
\end{tabular}

Tab. 1. $\mathrm{n}=$ nachleuchtend; Cla $=$ Clarophan $\left(\right.$ A u er); ${ }^{*}=$ gemessen bei $60^{\circ} \mathrm{C}$.

so ergeben sich Werte (s. Tab. 1, Spalte 4), die ein Maß für den Ausdruck $i_{0} /(C \cdot \alpha)$ darstellen, da der übrige Teil der Gl. (7) gleich 1 wird. Nun bedeutet aber $i_{0} / \alpha$ die gesamte, die Kapazität $C$ aufladende Elektrizitätsmenge, die durch den Leuchtprozeß erzeugt wird; $i_{0} / \alpha$ ist der gesamten auf der Photokathode des Vervielfachers erzeugten Elektronenzahl proportional. Wegen der Konstanz von $C$ ist daher das Verhältnis der $l$-Werte für $R=\infty$ ein Maß für die insgesamt bei einer Szintillation auf der Photokathode entstehende Ladungsmenge. Bestünde der gesamte Leuchtprozeß also nur aus einem exponentiellen Vorgang, so müßten diese Werte sich ebenso verhalten wie die entsprechenden Gleichstromwerte, die sich mit Hilfe eines Galvanometers am Ausgang des Vervielfachers messen lassen. Wir haben eine derartige Meßreihe aufgenommen und gefunden, daß diese Annahme keinesfalls zutrifft, sondern daß beträchtliche Abweichungen auftreten. Man erkennt in Spalte 5 der Tab. 1 , in der für Naphthalin der Ruhestromwert willkürlich gleich dem Wert von $l_{\infty}$ gesetzt ist, daß die Gleichstromwerte der verschiedenen Leuchtstoffe sich teilweise ganz anders verhalten als die entsprechenden Werte $l_{\infty}=i_{0} /(C \cdot \alpha)$.

Wir setzen voraus, daß beim Naphthalin der Leuchtprozeß nur aus dem exponentiellen Impulsvorgang besteht - was nach unseren früheren Messungen zwar als wahrscheinlich, aber nicht als sicher anzusehen ist - , wodurch die Gleichsetzung von $i_{0} /(C \cdot \alpha)_{\text {Naphth. }}$ und $I_{\text {Naphth. }}$ ihre physikalische Be. gründung erhält. Dann erkennt man aus den Werten der Tab. 1, daß alle übrigen Leuchtstoffe größere Gleichstromwerte ergeben, als es den $i_{0} /(C \cdot \alpha)$ Werten entspricht. Die Erklärung dieser Diskrepanz ist ziemlich naheliegend: Bei den meisten Leuchtstoffen wird nur ein Teil der durch $a$-Teilchen erzeugten Lichtenergie in Form des ersten, kurzzeitigen Leuchtens ausgestrahlt, während der andere Teil weit langsamer ausgesandt wird und deshalb bei der Impulsmessung nicht mehr beobachtet werden kann. Wie groß das Verhältnis beider Anteile ist, ergibt sich aus Spalte 6 der Tab. 1, die die Größe des kurzzeitig ausgestrahlten Lichtblitzes in Bruchteilen der insgesamt erzeugten Lichtintensität enthält. Man findet, daß besonders die Zinksulfide und $\mathrm{Zn}_{2} \mathrm{SiO}_{4}$ sowie $\mathrm{KBr}-\mathrm{Tl}$ nur einen kleinen Bruchteil ihrer gesamten Lichtmenge als kurzen Lichtimpuls aussenden, während die Stoffe $\mathrm{ZnO}, \mathrm{CaWO}_{4}$ und $\mathrm{MgWO}_{4}$ den Hauptteil sofort zur Ausstrahlung bringen. Sollte es sich zeigen, daß auch Naphthalin einen Teil seiner Gesamtlichtmenge langsam ausstrahlt, so würden die Zahlen der Spalte 6 entsprechend zu verkleinern sein.

Aus den Werten der Spalte 6 der Tab. 1 läßt sich auch der Prozentsatz der eingestrahlten $\alpha$-Energie errechnen, der als Lumineszenzimpuls ausgestrahlt wird. In einer früheren Arbeit ${ }^{5}$ wurden bereits die Absolutausbeuten $\eta$ der von uns untersuchten Leuchtstoffe bei Anregung mit $\alpha$-Teilchen angegeben; sie sind in Spalte 7 der Tab. 1 noch einmal zusammengestelit. Da diese Werte den insgesamt in Licht umgewandelten Bruchteil der $\alpha$-Teilchenenergie darstellen, kann man die Lichtausbeute $\eta_{l}$ des kurzzeitigen Prozesses aus den $l_{\infty} / I$-Werten bestimmen (Spalte 8 ). Setzt man die Lichtausbeute des Impulsvorganges bei Naphthalin willkürlich gleich 1, so lassen sich die Verhältnisse von $\eta_{l}$ für die verschiedenen Leucht-

${ }^{5}$ I. B roser, H. Ka $11 \mathrm{mann}$ u. U. M. Martius, Z. Naturforschg., 4a, 204 [1949]. 
stoffe leichter übersehen (Spalte 9). Die Werte geben dann auch gleichzeitig die Verhältnisse der Impulslängen $l_{\text {abs }}$ auf dem Oszillographen an, wenn mit einem Vervielfacher mit wellenlängenunabhängiger Elektronenausbeute der Photokathode gearbeitet würde.

\section{Diskussion}

Betrachtet man die in Tab. 1 wiedergegebenen Meßergebnisse, so fällt vor allem auf, daß die Abklingdauer des kurzzeitigen Leuchtprozesses von Leuchtstoff $\mathrm{zu}$ Leuchtstoff erstaunlich wenig variiert. Sieht man von dem bei $-60^{\circ} \mathrm{C}$ gemessenen $\alpha$-Wert von $\mathrm{CdS} \mathrm{ab}$, so liegen die Zeitkonstanten der untersuchten, in ihren sonstigen Eigenschaften sehr verschiedenartigen Leuchtstoffe innerhalb nur einer Zehnerpotenz. Das Interessante dabei ist, daß gerade Leuchtstoffe mit langem Nachleuchten die kürzesten Abklingzeiten aufweisen; so ist z. B. der in der Tab. 1 unter 4 angegebene Stoff ein $\mathrm{ZnS}-\mathrm{Cu}$ mit extrem guten Nachleuchteigenschaften (Clarophan der Firma Auer); er besitzt jedoch von allen untersuchten Substanzen die kurzzeitigsten Lichtblitze. Andererseits zeigt $\mathrm{CaWO}_{4}$, das bei Dauerbestrahlung momentan abgeklungen ist, ein besonders langzeitiges Abklingen.

Außer durch dieses kurzzeitige „Fluoreszenzleuchten“ können viele Leuchtstoffe Lumineszenzlicht auch nach viel längerer Zeit ausstrahlen. Besonders die Leuchtstoffe der Zink-Cadmium-Gruppe, $\mathrm{Zn}_{2} \mathrm{SiO}_{4}$ und $\mathrm{KBr}-\mathrm{Tl}$, zeichnen sich durch einen sehr hohen Prozentsatz langsam ausgestrahlter Lichtenergie aus. Nun besitzen diese Stoffe aber alle gute Phosphoreszenzeigenschaften, so daß man den langzeitigen Vorgang ganz einfach als Phosphoreszenz bezeichnen könnte. Dagegen sprechen aber zwei Tatsachen: Einmal ist bekannt und auch einfach nachzumessen, daß die Phosphoreszenz der Leuchtstoffe bei Anregung mit $\alpha$-Teilchen nur sehr schwer zu erregen ist, und zum anderen zeigt es sich, daß Leuchtstoffe mit guten Phosphoreszenzeigenschaften (Tab. 1, Nr. 3, 4, 7) sich gegenüber Leuchtstoffen des gleichen Typus mit geringer Phosphoreszenz (Tab. 1, Nr. 1, 2,6) nicht durch einen besonders hohen Anteil an langzeitigem Leuchten unterscheiden. Man muß also annehmen, daß die eigentliche Phosphoreszenz und das beobachtete langsame Abklingen nur sehr wenig miteinander gemein haben. Im allgemeinsten Fall dürfte also der Leuchtprozeß bei Anregung durch $\alpha$-Teilchen aus einem sehr kurzzeitigen exponentiellen Abklingen („Fluoreszenz"), einer längeren Abklingung (,momentane Phosphoreszenz“) und schließlich aus einer Minuten dauernden echten Phosphoreszenz bestehen. Es ist durchaus möglich, daß sich in einigen Fällen die beiden ersten Vorgänge durch ein einheitliches, hyperbolisches Gesetz beschreiben lassen. Wir glauben, daß die momentane Phosphoreszenz schon nach einer relativ kurzen Zeit beendet ist. Betrachtet man nämlich den Abklingvorgang eines Impulses auf dem Oszillographenschirm mit stärkerer zeitlicher Auflösung bei kleinerer Zeitkonstante, so beobachtet man einen um so stärker gezackten, unregelmäßigen Kurvenverlauf, je größer der Anteil an langzeitiger Lumineszenz am betreffenden Leuchtstoff ist. Dieser Vorgang zeigt deutlich, daß ein groBer Anteil an momentaner Phosphoreszenz in Zeiträumen zur Ausstrahlung gelangt, die zwar groß sind gegenüber der Fluoreszenzzeit, aber klein gegenüber Zeiten, in denen normalerweise die Phosphoreszenz ausgestrahlt wird. Aus der Beobachtung des gezackten Verlaufs der Oszillographenkurve kann man erkennen, daß in etwa $10^{-4}$ bis $10^{-2}$ sec praktisch die gesamte momentane Phosphoreszenz beendet ist.

Interessant ist der Vergleich der Abklingzeiten von Naphthalin bei Anregung mit $\alpha$ - und $\beta$-Teilchen. Wie wir nach der gleichen Methode feststellen konnten, liegt die Abklingkonstante bei Erregung mit $\beta$ oder $\gamma$-Strahlen um etwa eine Größenordnung höher als bei Erregung mit $\alpha$-Teilchen. Bemerkt sei noch, daß die Abklingzeit des $\beta$-Impulses gut mit den von G. B. Coll in s ${ }^{6}$ gefundenen Werten übereinstimmt. Beinahe umgekehrt ist es bei den Zink-CadmiumSulfiden und beim KBr: Hier überwiegt bei Erregung mit $\beta$-Strahlen der Anteil der momentanen Phosphoreszenz und der Phosphoreszenz derart, daß kurzzeitige Impulse nur schwer zu beobachten sind. Man kann also sagen, daß die $\alpha$-Impulse bei diesen Stoffen kurzzeitiger sind als die $\beta$-Impulse. Genaue Messungen über die Vorgänge bei $\beta$-Erregung an allen untersuchten Leuchtstoffen stehen noch aus ${ }^{7}$.

Die durchgeführten Messungen sind von Wichtigkeit für die Anwendung des Leuchtmassenzählers zur Registrierung von $\alpha$-Teilchen. Sie zeigen nämlich. daß die Stoffe mit den größten Lichtblitzen - also die Zink-Cadmium-Sulfide — sehr wohl zur Zählung sehr vieler Teilchen, beispielsweise in einer Koinzidenzanordnung, angewendet werden können. Wählt man die zur Registrierung benutzte Verstärkeranordnung mit genügend großer Zeitkonstante, so stört der Vorgang der momentanen Phosphoreszenz nur noch sehr wenig.

6 G. B. Collin s, Physic. Rev. 74, 1543 [1948].

- R. H o f s t äd te r, Physic. Rev. 75 [1949]. 
Zur theoretischen Erklärung unserer Messungen bedarf es unserer Meinung nach noch einer großen Zahl weiterer Versuche. Vor allem muß die Meßgenauigkeit erhöht werden, wodurch die Entscheidung zwischen exponentiellem und hyperbolischem Verlauf dann sicherer als bisher getroffen und der Zahlenwert für $\alpha$ mit kleinerem Fehler angegeben werden kann. Weiterhin dürften Messungen über das Spektrum des Fluoreszenzanteiles im Verhältnis zum
Spektrum der länger dauernden Vorgänge von Wichtigkeit sein. Schließlich ließen sich auf Grund von Untersuchungen über den Einfluß der Temperatur und der Korngröße der Leuchtstoffe wichtige Aufschlüsse über die Vorgänge, die das Auftreten der drei beschriebenen Leuchtprozesse verursachen, geben. Derartige Versuche sind bei uns im Gange ${ }^{8}$.

${ }^{8}$ Frl. I. K la mpt und Hrn. G. Gold b a ch danken wir für Mitarbeit bei der Durchführung der Messungen.

\title{
Der Massendefekt als Folge der relativistischen Feldgleichung für das Zweikörperproblem
}

\author{
Von Walter Thirring * \\ (Z. Naturforschg. 5 a, 85-88 [1950]; eingegangen am 10. Juli 1949)
}

\begin{abstract}
Es wird die relativistische Feldgleichung für zwei Partikel mit Spin $1 / 2$ aufgestellt und daraus deduziert, daß sie sich, falls sie untereinander gebunden sind, verhalten wie ein Teilchen, dessen Masse gleich der Summe der Massen vermindert um die Bindungsenergie ist.
\end{abstract}

$D^{e}$ er Massendefekt ist eine der besten empirischen Stützen der Relativitätstheorie und ein Grundpfeiler der gesamten Kernphysik. Aus den Aussagen der Relativitätstheorie folgt unmittelbar, daß zwei Teilchen, die untereinander durch eine Kraft verbunden sind, sich in einem äußeren Kraftfeld tatsächlich so verhalten wie ein Teilchen mit der Summe der Massen minus der Bindungsenergie als Masse. Trotzdem ist es im einzelnen oft schwierig, dieses Ergebnis der Relativitätstheorie nachzurechnen, da die Rechnung die Lösung des relativistischen Mehrkörperproblems voraussetzt. Strenge Lösungen gibt es bisher nicht. Es existieren nur Näherungslösungen, die um eine Näherung weitergehen als der klassische Standpunkt. Im Rahmen dieser Näherung konnte auch B a g g e ${ }^{1}$ zeigen, daß der Massendefekt aus einer Lagrange-Funktion folgt, welche der bekannten Darwinschen relativistischen Lagrange-Funktion nachgebildet ist. Die Arbeit von Bagge verwendet jedoch die klassische Partikeltheorie, und es scheint geboten, zu untersuchen, ob die angemessene feldmäßige Behandlung der Elementarpartikel dasselbe leistet. Auch scheinen die Grundlagen der Baggeschen Arbeit nicht willkürfrei, da er seine Lagrange-Funktion nur dadurch propagieren kann, daß sie im Fall des Coulombschen Kraftgesetzes in die Darwinsche übergeht. In dieser Arbeit soll nun

* Wien IX, Strudlhofgasse 13.

1 E. B a g g e, Z. Naturforschg. 1, 361 [1946]. gezeigt werden, daß aus der relativistischen Feldgleichung für das Zweikörperproblem nach Reduktion auf die großen Komponenten und Separation tatsächlich für die Schwerpunktskoordinaten eine Schrödinger-Gleichung folgt, in welcher als Masse die Summe der Massen minus der Bindungsenergie steht. Auch wird hier der Willkür kein Spielraum gelassen, da die Feldgleichung durch die Forderung der relativistischen Invarianz eindeutig bestimmt wird. Dabei stellt sich auch heraus, daß die bisher verwendete Feldgleichung für das Zweikörperproblem nicht streng invariant ist und daher einen falschen Massendefekt liefert.

\section{$\S 1$. Aufstellung der Feldgleichung}

Eine relativistische invariante Feldgleichung für zwei Partikel mit Spin 1/2 muß im kräftefreien Fall folgende Gestalt haben:

$$
\left(\gamma_{1}^{i} \frac{\partial}{\partial x_{i}{ }^{1}}+\varkappa_{2}^{i} \frac{\partial}{\partial x_{i}{ }^{2}}+\mu_{1}+\mu_{2}\right) \psi^{\prime}\left(x^{1}, x^{2}\right)=0 .
$$

Hier steilen die $\gamma$ die üblichen Diracschen Matrizen dar, $\mu$ bedeutet die reziproke Compton-Wellenlänge, und die Indizes 1 und 2 beziehen sich auf die Partikel. Auch wollen wir im folgenden die jetzt üblichen Fundamentalgrößen verwenden, als Masse $m c^{2}$, als Impuls $p c$ und als Wirkungsquantum $h c / 2 \pi$. Wenn wir nun in Gl. (1) wie gewöhnlich die Substitution 